\title{
Prevalence and risk factors of latent Tuberculosis among adolescents in rural Eastern Uganda
}

\author{
Daniel Mumpe-Mwanja ${ }^{1,2,3,4}$, Suzanne Verver ${ }^{5}$, Adoke Yeka ${ }^{6}$, Alfred Etwom ${ }^{4}$, James Waako ${ }^{2,3}$, \\ Willy Ssengooba ${ }^{7,3}$, Joseph KB Matovu ${ }^{1}$, Rhoda K Wanyenze ${ }^{1,6}$, Phillipa Musoke ${ }^{3,8}$, \\ Harriet Mayanja-Kizza ${ }^{9,3}$
}

1. Makerere University School of Public Health, CDC fellowship program

2. Makerere University, Iganga Mayuge Health Demographic Surveillance Site

3. Makerere University College of Health Sciences, Infectious Diseases Institute

4. Ministry of Health, National Tuberculosis and Leprosy Program

5. KNCV Tuberculosis Foundation; CINIMA, Academic Medical Centre

6. Makerere University School of Public Health, Department of Disease control and Environmental Health

7. Makerere University School of Medicine, Department of Microbiology

8. Makerere University School of Medicine, Department of Pediatrics and Child Health

9. Makerere University School of Medicine, Department of Medicine

\begin{abstract}
Background: Latent Tuberculosis treatment is a key tuberculosis control intervention. Adolescents are a high risk group that is not routinely treated in low income countries. Knowledge of latent Tuberculosis (TB) burden among adolescents may influence policy.

Objectives: We determined the prevalence and risk factors of latent TB infection among adolescents in rural Uganda.

Methods: We analyzed baseline data from a study that assessed the prevalence and incidence of Tuberculosis disease among adolescents. We extracted socio-demographics, medical assessment information, and tuberculin skin test results and estimated prevalence ratios (PR) of latent TB infection risk factors by binomial regression.

Results: The prevalence of latent TB was $16.1 \%, 95 \%$ CI $(15.1$ - 17.2). Significant risk factors were: a BCG scar, APR 1.29 (95\% CI 1.12 - 1.48); male gender, APR 1.37 (95\% CI 1.21 - 1.56); age 17 -18 years, APR 1.46 (95\% CI 1.24 - 1.71) and 15-16 years, APR 1.25 (95\% CI 1.07 - 1.46) compared to 12-14 years; being out of school, APR 1.31 (95\% CI 1.05 - 1.62); and a known history of household TB contact in last 2 years, APR 1.91 (95\% CI $1.55-2.35)$

Conclusion: Targeted routine latent TB treatment among adolescents out of school may be crucial for TB disease control in low income countries.

Keywords: Latent tuberculosis infection, Adolescents, Risk factors, Tuberculin skin testing, Tuberculosis

DOI: http://dx.doi.org/10.4314/ahs.v15i3.20

Cite as: Mumpe-Mwanja D, Verver S, Yeka A, Etwom A, Waako J, Ssengooba W, Matovu JKB, Wanyenze RK, Musoke P, Mayanja-Kizza H. Prevalence and predictors of latent Tuberculosis among adolescents in rural Eastern Uganda. Afri Health Sci. 2015;15(3):85160. doi: http:// dx.doi.org/10.4314/abs.v15i3.20

\section{Introduction}

Tuberculosis (TB) is one of the commonest infectious

\author{
Corresponding author: \\ Daniel Mumpe-Mwanja, \\ Makerere University School \\ of Public Health, \\ CDC fellowship program; \\ Makerere University, Iganga Mayuge \\ Health Demographic Surveillance Site; \\ Makerere University College of Health Sciences, \\ Infectious Diseases Institute; \\ Ministry of Health, National \\ Tuberculosis and Leprosy Program
}

Email: mwanjamumpe@yahoo.com

diseases in the world. In 2012 the estimated global burden of disease was 8.6 million cases ${ }^{1}$. Considerable TB control success has been registered through interruption of disease transmission. This has been achieved by identifying and treating persons with the disease ${ }^{2,3}$. Following infection with TB, an individual's immunity can suppress TB organisms to a Latent TB infection (LTBI) state. Reactivation may occur many years after infection leading to active disease and has been documented as an important source of new active disease $e^{4}$. Whereas high income countries have incorporated the diagnosis and treatment of latent TB infection among all individuals in their TB control programs, low income countries usually target this intervention for only HIV infected patients and children with a household TB contact ${ }^{5}$, the groups at highest risk of LTBI reactivation.
\end{abstract}


There is a sudden increase of TB incidence during adolescence, which may be due to an increase in new infections ${ }^{6}$ or following conversion of LTBI to active disease. This makes adolescence suitable for targeted TB disease control interventions that prevent progression of LTBI to active disease. Few studies have documented the prevalence and risk factors of latent $\mathrm{TB}$ infection among adolescents ${ }^{7-9}$. We assessed the prevalence of latent TB infection, and it's risk factors among adolescents in and out of school in two rural district of Eastern Uganda. Knowledge of the epidemiology of latent TB in this age group is important to guide the design of control interventions.

\section{Methods}

\section{Study design}

We reviewed baseline data for participants enrolled in a prospective cohort study conducted among adolescents to determine the prevalence and incidence of TB disease in the Makerere University School of Public Health Demographic Surveillance Site (DSS) in Eastern Uganda ${ }^{10}$.

\section{Enrolment and baseline assessment for main study} Details of the main study are published elsewhere ${ }^{11}$. The main study enrolled adolescents aged 12-18 years who had resided in the study area for at least three months prior to the study and had no plans of migrating were recruited into the main study cohort. A total of 5000 adolescents were proportionately enrolled from three age strata (12-14, 14-16 and 17-18 years) and followed-up for at least one year.

Enrolled study participants were assessed on two occasions either at their residence or school by study nurses. At the first assessment, a full medical history and focused physical examination was done. Information was collected on socio-demographics, medical history pertaining to signs and symptoms of TB disease, history of household contact with a TB patient within two years prior to enrolment in the study and history of smoking, alcohol consumption and hospitalization within the last six months. Physical examination included inspection of the BCG vaccination site for a scar and TB specific signs. All participants had a tuberculin skin test (TST) performed by sub-dermal administration of two units of tuberculin / purified protein derivative (PPD) from the Staten's Serum Institute, Denmark on the volar aspect of the right forearm using the mantoux technique. Though it was desirable to perform an HIV test on all participants, this was not done due to community concerns. Only participants who had prevalent TB had an HIV test performed.

A second assessment visit was done between 48 and 72 hours after the TST, during which the TST site was examined for an induration. The transverse diameter of the induration at the site was measured using a transparent ruler and recorded in millimeters $(\mathrm{mm})$. All baseline procedures were performed under adequate quality control measures by study nurses who had undergone study tailored training.

\section{Interpretation of TST readings}

TST readings were used to determine whether a participant had LTBI or not. A participant was said to have LTBI if they had a positive TST. A TST was considered positive if; the transverse skin induration diameter was greater than or equal to $5 \mathrm{~mm}$ in an HIV infected participant, and greater than or equal to $10 \mathrm{~mm}$ in a participant without HIV infection.

\section{Eligibility criteria for this study}

Baseline data of all participants enrolled in the main study was eligible for inclusion in this study. Data of participants whose skin induration diameter was not measured between 48-72 hours of TST administration were excluded.

\section{Data management}

Baseline data of all participants enrolled in the main study was extracted from the Microsoft SQL Server 2008 database into Microsoft Excel 2007 for cleaning. Cross tabulation and calculation of frequencies were done using pivot tables to further edit and clean the data. A data dictionary was also developed to describe values in the variables.

\section{Data analysis}

Clean data was then exported to SPSS version 16 for univariate analysis. To describe the characteristics of the study population, we calculated the frequencies of the socio-demographic and predictor variables i.e. age, gender, schooling status, smoking habit, drinking habit, hospitalization history in the six months prior to the study, HIV sero-status, presence of a visible BCG scar and history of household contact with a TB patient in the last two years. We plotted the distribution of TST reading diameters and later categorized the readings as either positive or negative. We calculated the frequency 
of baseline positive tuberculin skin test to determine the prevalence of LTBI.

Data was then exported to STATA version 12 to determine the risk factors of prevalent LTBI. We used a mixed effects binomial model with a logarithmic link function to estimate prevalence ratios (PR) for risk factors of latent tuberculosis infection (LTBI).

\section{Ethical consideration}

The study was approved by the Makerere University School of Public Health-Higher Degrees and Research Ethics committee (HDREC), Kampala Uganda and the Uganda National Council of Science and Technology (UNCST). Written informed consent was obtained from the parents or legal guardians of the study participants and the adolescents provided assent.

Table 1: Characteristics of study population

\section{Results}

\section{Enrolment of participants}

A total of $4981(99.5 \%)$ out of the 5000 adolescents in the main study were included in the analysis for this study. We excluded 19 participants $(0.38 \%)$ from the analysis because either they were not available for measurement or the measurement was not done within the study stipulated $48-72$ hours after TST administration.

\section{Characteristics of participants}

Overall, $2332(46.8 \%)$ of the study participants were 12-14 years old, $1475(29.6 \%) 15-16$ year old and 1174 $(23.6 \%)$ were $17-18$ years old A total of $4663(93.6$ $\%)$ participants were out of school, $235(4.72 \%)$ had a known history of a household TB contact within two years prior to enrolment, $3230(64.8 \%)$ had a visible BCG scar; and six participants $(0.12 \%)$ were HIV infected. Details of the participant characteristics are summarized in Table 1.

\begin{tabular}{|c|c|c|}
\hline $\begin{array}{l}\text { Variable } \\
\text { Age stratum }\end{array}$ & Frequency $(n=4981)$ & Proportion (\%) \\
\hline $12-14$ years & 2332 & 46.8 \\
\hline $15-16$ years & 1475 & 29.6 \\
\hline $17-18$ years & 1174 & 23.6 \\
\hline \multicolumn{3}{|l|}{ Gender } \\
\hline Male & 2582 & 51.9 \\
\hline \multicolumn{3}{|c|}{ Schooling status } \\
\hline In school & 4663 & 93.6 \\
\hline \multicolumn{3}{|l|}{ Smoking habit } \\
\hline Yes & 2 & 0.04 \\
\hline \multicolumn{3}{|l|}{ Drinking habit } \\
\hline Yes & 11 & 0.22 \\
\hline \multicolumn{3}{|c|}{ History of hospitalization in last 6 months } \\
\hline Yes & 43 & 0.86 \\
\hline \multicolumn{3}{|c|}{ HIV sero-status } \\
\hline HIV infected & 4 & 0.08 \\
\hline \multicolumn{3}{|c|}{ Presence of BCG scar } \\
\hline Yes & 3230 & 64.8 \\
\hline \multicolumn{3}{|c|}{ Known history of household contact with TB patient in last 2 years } \\
\hline Yes & 235 & 4.72 \\
\hline
\end{tabular}


Prevalence of latent TB infection

The overall prevalence of latent TB infection (LTBI) was $16.1 \%$, (95\% confidence interval (CI) $15.1-17.2)$.
The distribution of TST induration diameters showed little digit preference, indicating good quality reading; and showed a bimodal distribution with 2 peaks, at 6-8 $\mathrm{mm}$ and $12 \mathrm{~mm}$ (Figure 1).

\section{Figure 1: Distribution of TST induration diameter among the study participants $\infty$}

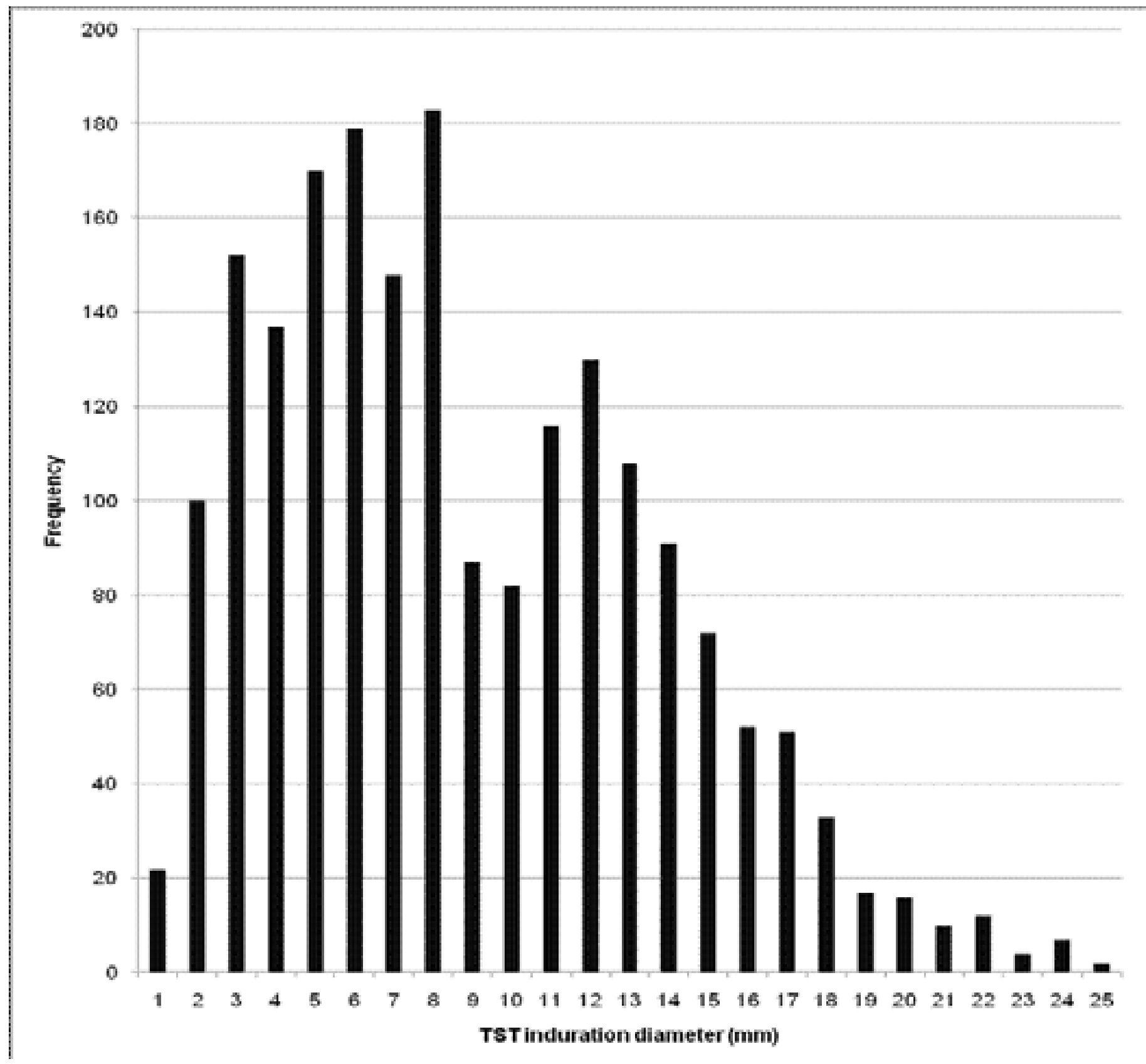

$\infty$ We excluded 3000 participants with zero TST induration diameters; this graph includes TST readings for 1981 participants.

The prevalence of LTBI increased progressively by age stratum from $37 \%$ in the $12-14$ years stratum to $41 \%$ in the 15-16 strata and 47\% in the 17-18 age stratum (Figure 2). 
Figure 2: Distribution of LTBI (positive TST) within the age strata.

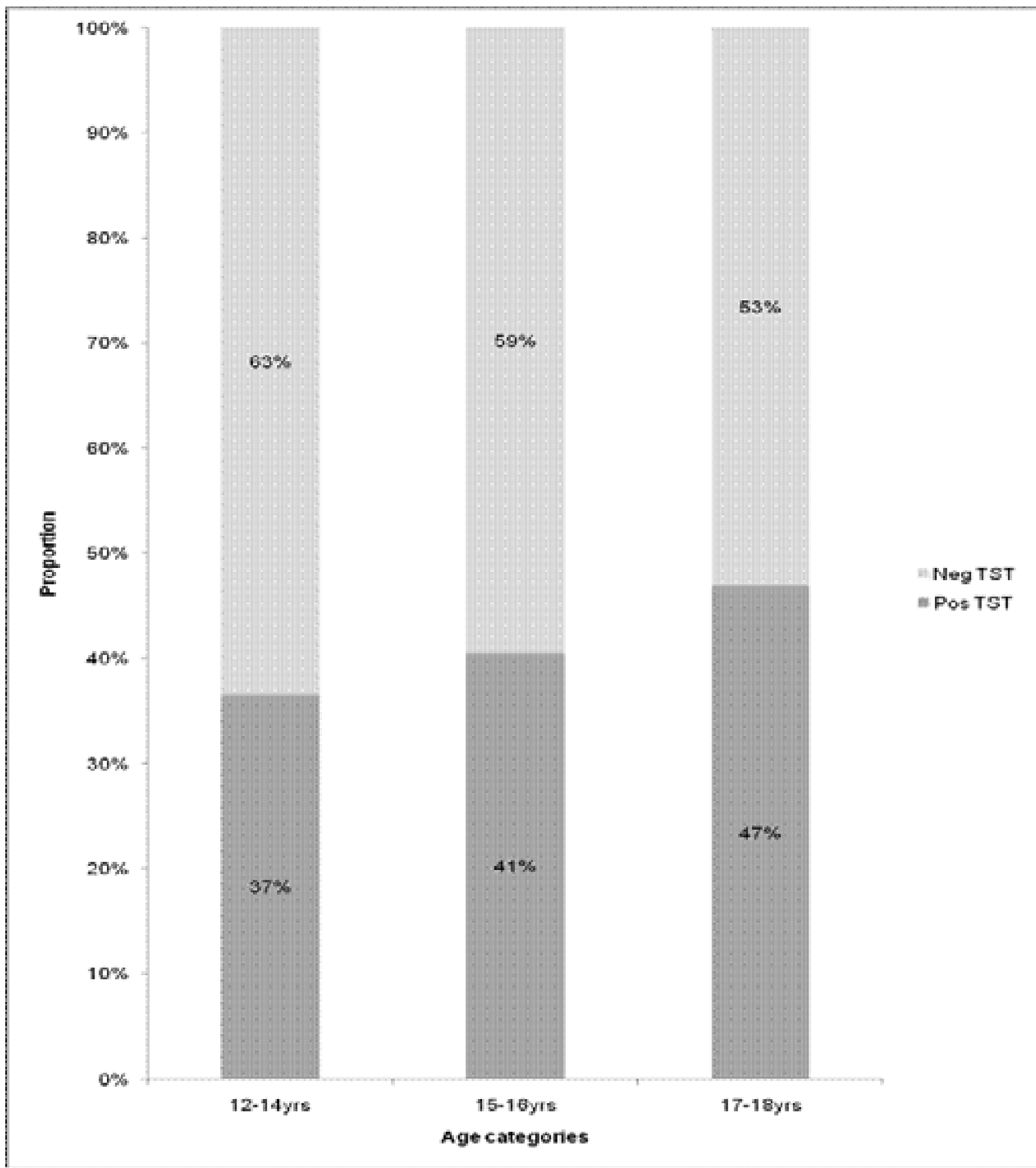

Risk factors of latent TB infection

At bivariate analysis: a BCG scar, Male gender, age strata $15-16$ years, $17-18$ years, non-school going status, and having no household history of contact with a TB patients had significant association with LTBI among adolescents (Table 2). 
Table 2: Bivariate analysis of association between LTBI and participant characteristics $(n=4981)$

\begin{tabular}{|c|c|c|c|c|}
\hline Characteristic & Participants (N) & Participants with LTBI n (\%) & Crude PR (95\% Cl) & p-value \\
\hline \multicolumn{5}{|l|}{ TST Result } \\
\hline Positive & 4981 & $803(16.1)$ & $16.1(15.1-17.2)$ & \\
\hline \multicolumn{5}{|l|}{ BCG scar } \\
\hline No & 1751 & $233(13)$ & 1 & \\
\hline Yes & 3230 & $570(18)$ & $1.33(1.15-1.53)$ & $<0.001$ \\
\hline \multicolumn{5}{|c|}{ Hospitalization history } \\
\hline No & 4938 & $796(16)$ & 1 & \\
\hline Yes & 43 & $7(16)$ & $1.01(0.51-1.99)$ & 0.977 \\
\hline \multicolumn{5}{|c|}{ Alcohol Consumption } \\
\hline No & 4970 & $802(16)$ & $1.77(0.27-11.51)$ & 0.548 \\
\hline Yes & 11 & $1(9)$ & 1 & \\
\hline \multicolumn{5}{|l|}{ Gender } \\
\hline Female & 2399 & $322(13)$ & 1 & \\
\hline Male & 2582 & $481(19)$ & $1.39(1.22-1.58)$ & $<0.001$ \\
\hline \multicolumn{5}{|l|}{ Age strata } \\
\hline $12-14$ & 2332 & $308(13)$ & 1 & \\
\hline $15-16$ & 1475 & $248(17)$ & $1.27(1.09-1.48)$ & 0.002 \\
\hline $17-18$ & 1174 & $247(21)$ & $1.59(1.37-1.85)$ & $<0.001$ \\
\hline \multicolumn{5}{|c|}{ Schooling status } \\
\hline In school & 4663 & $726(16)$ & 1 & \\
\hline Out of school & 318 & $77(24)$ & $1.55(1.27-1.91)$ & $<0.001$ \\
\hline \multicolumn{5}{|c|}{ Household TB contact } \\
\hline \multicolumn{5}{|l|}{ history } \\
\hline No & 4746 & $734(15)$ & 1 & \\
\hline Yes & 235 & $69(29)$ & $1.90(1.54-2.34)$ & $<0.001$ \\
\hline \multicolumn{5}{|l|}{ HIV infection } \\
\hline No or Unknown & 4975 & $801(16)$ & 1 & \\
\hline Infected & 6 & $2(33)$ & $2.07(0.67-6.43)$ & 0.208 \\
\hline
\end{tabular}

At multivariable analysis, the binomial regression model was significant $(\mathrm{p}=0.002)$. Participants with a visible BCG scar (previous BCG vaccination), APR 1.29 (95\% CI 1.12 - 1.48, p<0.001); male gender, APR 1.37 (95\% CI $1.21-1.56, \mathrm{p}<0.001)$; age strata $17-18$ years, APR $1.46(95 \%$ CI $1.24-1.71, \mathrm{p}<0.00115-16$ years, APR
$1.25(95 \%$ CI $1.07-1.46) \mathrm{p}=0.004$, and ; being out of school, APR 1.31 (95\% CI 1.05 - 1.62, p< 0.014); having a known history of household TB contact in last 2 years, APR $1.91(95 \%$ CI $1.55-2.35, \mathrm{p}<0.001)$ were significant risk factors for latent TB infection among the adolescents (Table 3). 


\begin{tabular}{|c|c|c|c|c|}
\hline Characteristic & Crude PR (95\% Cl) & p-value & Adjusted PR $(95 \% \mathrm{Cl})$ & p-value \\
\hline \multicolumn{5}{|l|}{ BCG scar } \\
\hline No & 1 & & 1 & \\
\hline Yes & $1.33(1.15-1.53)$ & $<0.001$ & $1.29(1.12-1.48)$ & $<0.001$ \\
\hline \multicolumn{5}{|l|}{ Gender } \\
\hline Female & 1 & & 1 & \\
\hline Male & $1.39(1.22-1.58)$ & $<0.001$ & $1.37(1.21-1.56)$ & $<0.001$ \\
\hline \multicolumn{5}{|l|}{ Age strata } \\
\hline $12-14$ & 1 & & 1 & \\
\hline $15-16$ & $1.27(1.09-1.48)$ & 0.002 & $1.25(1.07-1.46)$ & 0.004 \\
\hline $17-18$ & $1.59(1.37-1.85)$ & $<0.001$ & $1.46(1.24-1.71)$ & $<0.001$ \\
\hline \multicolumn{5}{|c|}{ Schooling status } \\
\hline In school & 1 & & 1 & \\
\hline Out of school & $1.55(1.27-1.91)$ & $<0.001$ & $1.31(1.05-1.62)$ & 0.014 \\
\hline $\begin{array}{l}\text { Household TB } \\
\text { contact histor }\end{array}$ & & & & \\
\hline No & 1 & & 1 & \\
\hline Yes & $1.90(1.54-2.34)$ & $<0.001$ & $1.91(1.55-2.35)$ & $<0.001$ \\
\hline
\end{tabular}

\section{Discussion}

In this study conducted in a rural based Iganga-Mayuge demographic surveillance site, we found a $16.1 \%$ prevalence of LTBI among adolescents aged 12-18 years. The risk factors of prevalent LTBI were: age strata 17 - 18 and 16 -17 years, male gender, a visible BCG scar, a known history of household TB contact within the last 2 years and being out of school.

The LTBI prevalence of $16.1 \%$ is quite low in comparison to the $42.2 \%$ prevalence found among adolescents in a South African study where positive TST was defined as TST induration diameter cut off of $10 \mathrm{~mm}^{8}$.
This is could be due to the higher TB incidence rate in South Africa; 857 per 100,000 population compared to Uganda's 175 per 100,000 population'. Given that latent TB is a big source of new active disease ${ }^{4}$, a higher incidence of TB disease in South Africa suggests a similarly higher LTBI prevalence than Uganda. Furthermore, the lower LTBI prevalence found in our study may be related to the rural nature of the study population that is associated with a lower risk of TB transmission compared to the more urban setting in which the South Africa study was conducted ${ }^{8,10,11}$.

Use of two-step TST administration has been found to increase LTBI detection; an increase of $11 \%$ TST 
positivity was found when two-step TST was used in a recent study conducted in two Columbian prisons ${ }^{12}$, but only $2 \%$ increase in LTBI in Indian adolescents ${ }^{13}$. A two-step TST administration was not feasible in our community based study and we may thus have underestimated the LTBI prevalence in our study population. Older age was associated with a higher risk of LTBI similar to previous studies involving students ${ }^{7,8}$. This is due to a larger cumulative exposure to patients with active TB disease with increasing age, as has been observed in high prevalence countries ${ }^{14,15}$.

Though schools offer a congregate environment ideal for transmission of respiratory infections, we found a higher proportion of adolescents with LTBI among those out of school than those in school. The adolescents out of school are more likely to engage in smoking and alcohol consumption which have been found to increase the risk for TB infection ${ }^{16,17}$, although we could not confirm that in this study, possibly due to low smoking and alcohol consumption in the study population.

The prevalence of LTBI among the male participants was higher than that in the female participants. This is no surprise since the global TB disease burden is higher among males than females ${ }^{1}$. A larger TB disease prevalence among males compared to females was also found in disease surveys conducted in some high prevalence countries like Malawi and Nigeria. This may be attributed to the higher LTBI prevalence in males and/or higher reactivation rate among the males and our study seems to indicate the former.

We found a significant association between previous BCG vaccination and prevalent LTBI in contrast to other studies ${ }^{8,18}$. This could be due to a false positive LTBI arising from the BCG reaction with the TST, the sole investigation that our study used to determine LTBI. The effect of BCG on TST reactivity has however been shown to wane off after 8-10 years ${ }^{19,20}$. In Uganda BCG is given at birth, therefore a positive TST among adolescents is more likely to reflect true LTBI. However, TST reactivity to BCG has also been demonstrated long after the period that the effect of the BCG vaccination is expected to have waned off ${ }^{20}$, which may explain why our study found more LTBI among adolescents who had a BCG scar (BCG vaccination). Though the demonstration of TST reactivity to BCG vaccination creates controversy about the use of TST to measure LTBI in countries with high BCG coverage, such as
Uganda $^{21,22}$, studies that used interferon gamma release assays (IGRA's), that are believed not to be affected by BCG have shown no added value in estimation of LTBI in high BCG vaccination areas ${ }^{7,8,22}$.

The strength of this study included the use of clinical history coupled with microbiological and radiological investigations to exclude active TB disease among all participants. We were thus able to identify and exclude participants with active TB disease from our analysis to provide a more accurate estimate of LTBI and its risk factors. The study also enrolled both school going and out of school adolescents, the findings of this study therefore give information that can be used to make disease control interventions that are applicable to both categories of adolescents.

\section{Limitations}

Our inability to differentiate and subsequently exclude from analysis positive TST reactions due to non-tuberculous mycobacterium (NTM) infection. However the TST distribution diameter had two peaks, with the first peak at an induration diameter ranging between 6-8 $\mathrm{mm}$; this first peak most likely represent NTM. The TST positivity cut-off of $10 \mathrm{~mm}$ could have largely excluded these reactions due to NTM.

In our study the BCG scar was used as a proxy to measure BCG vaccination. However, the use of the scar may not be a reliable proxy, since a part of children who receive BCG vaccination may not develop a $\mathrm{scar}^{23,24}$.

The TST induration we used to determine LTBI is a cell mediated immune reaction which may be compromised by HIV infection ${ }^{25}$. To reduce false negative TST results among HIV infected persons a TST induration cut off diameter of $5 \mathrm{~mm}$ is recommended ${ }^{26,27}$. In this study, we failed to obtain consent for HIV testing and so we did not ascertain HIV status of all participants yet we used a TST induration diameter of $10 \mathrm{~mm}$ as a cut off to determine LTBI on all participants and so we may have underestimated the LTBI prevalence in our study population. However, more than $50 \%$ of our study population included adolescents aged $15-18$ years, an age group with a very low HIV prevalence $(1.4 \%)$, based on the most recent HIV sero-behavioral survey in Uganda ${ }^{28}$. More so, we expect an even lower HIV prevalence in those aged $12-14$ years $^{29}$. Thus, HIV infection may not have considerably affected the LTBI prevalence estimates. 


\section{Conclusion}

Our findings in these rural Eastern Uganda districts of Iganga and Mayuge show that BCG immunization (BCG scar) is significantly associated with TST positivity during adolescence. Latent TB is significantly higher among the non-school going adolescents and those with a household contact, even after correction for age and sex. Routine diagnosis and treatment of Latent TB targeting adolescents out of school may be a crucial intervention for TB disease control in low income countries.

\section{Conflict of interest}

There is no conflict of interest regarding the publication of this paper.

\section{Acknowledgements}

We are very grateful to the community of Iganga and Mayuge districts, especially the study participants for accepting to take part in the study, we are also grateful to the study staff for the immeasurable effort they put into the different study activities. We thank Anne Wajja and Patrick Nabongo for the organizational, logistic and administrative support they offered to the main study, Ronald Mutunzi for managing all the study data and Nico Nagelkerke for supporting us to analyse the data. We thank the European and Developing Countries Clinical Trials Partnership (EDCTP) for the funding the main study, grant CT.2005.32090.003, that generated the data used to write this paper.

\section{Disclaimer}

The contents of this article are solely the responsibility of the authors and do not necessarily represent the official views of the Centers for Disease Control and Prevention nor the National Tuberculosis and Leprosy program.

\section{Authors' contributions}

DMM: led the analysis and writing of the manuscript; SV: Participated in data analysis, critical review and writing of the manuscript, development of the main study protocol and was part of the team that conceived the papers written from data it generated; PM and HMK: participated in critical review and writing of the manuscript, development of the main study protocol, were part of the team that conceived the papers written from the data the main study generated and were principal investigators for the main study. AY, AE, JW, WS, JKBM and RKW: participated in critical review and manuscript writing.

\section{References}

1. WHO. Global tuberculosis report 2013. 2013.

2. WHO. Global tuberculosis report 2012. 2012.

3. Raviglione MC. The Global Plan to Stop TB, 20062015. Int J Tuberc Lung Dis. 2006;10(3):238-9.

4. Rowland R, McShane $H$. Tuberculosis vaccines in clinical trials. Expert Rev Vaccines. 2011;10(5):645-58.

5. WHO. TB/HIV Clinical Manual. Second ed. Geneva: WHO 2012.

6. Mandalakas AM, Starke JR. Current concepts of childhood tuberculosis. Semin Pediatr Infect Dis. 2005;16(2):93-104.

7. Dagnew A, Hussein J, Abebe M, Zewdie M, Mihret A, Bedru A, et al. Diagnosis of latent tuberculosis infection in healthy young adults in a country with high tuberculosis burden and BCG vaccination at birth. BMC Research Notes. 2012;5(1):415 PubMed .

8. Mahomed H, Hawkridge T, Verver S, Geiter L, Hatherill M, Abrahams DA, et al. Predictive factors for latent tuberculosis infection among adolescents in a high-burden area in South Africa. Int J Tuberc Lung Dis. 2011;15(3):331-6.

9. Hafizi H, Aliko A, Sharra E, Fico A, Migliori GB, Castiglia $\mathrm{P}$, et al. Results of a tuberculin skin testing survey in Albania. J Infect Dev Ctries. 2014;8(3):310 PubMed -4 .

10. HDSS IM. 2014 [20 September]. Available from: www.igangamayuge- hdss.mak.ac.ug/.

11. Waako J, Verver S, Wajja A, Ssengooba W, Joloba ML, Colebunders R, et al. Burden of tuberculosis disease among adolescents in a rural cohort in Eastern Uganda. BMC Infect Dis. 2013;13:349.

12. Rueda ZV, Arroyaye L, Marin D, Lopez L, Keynan Y, Giraldo MR, et al. High prevalence and risks factors associated with latent tuberculous infection in two Colombian prisons. Int J Tuberc Lung Dis. 2014 Oct;18(10):1166-72. doi 10.5588/ijtld.14.0179.

13. Murthy M, Selvam S, Jesuraj N, Bennett S, Doherty M, Grewal HM et al. Two-step tuberculin skin testing in school-going adolescents with initial 0-4 millimeter responses in a high tuberculosis prevalence setting in South India. PLoS One. 2013 Sep 6;8(9):e71470.

14. Lienhardt C, Fielding K, Sillah J, Tunkara A, Donkor S, Manneh K, et al. Risk factors for tuberculosis infection in sub-Saharan Africa: a contact study in The Gambia. Am J Respir Crit Care Med. 2003;168(4):448-55. 15. Roelsgaard E, Iversen E, Blocher C. Tuberculosis in Tropical Africa. An Epidemiological Study. Bull World Health Organ. 1964;30:459-518.

16. Leung CC, Yew WW, Law WS, Tam CM, Le- 
ung M, Chung YW, et al. Smoking and tuberculosis among silicotic patients. European Respiratory Journal. 2007;29(4):745-50.

17. Legesse M, Ameni G, Mamo G, Medhin G, Bjune G, Abebe F. Community-based cross-sectional survey of latent tuberculosis infection in Afar pastoralists, Ethiopia, using QuantiFERON-TB Gold In-Tube and tuberculin skin test. BMC Infect Dis. 2011;11:89.

18. Anderson R, Sy F, Thompson S, Addy C. Cigarette smoking and tuberculin skin test conversion among incarcerated adults. Am J Prev Med. 1997;13:175 - 81 PubMed .

19. Wang L, Turner MO, Elwood RK, Schulzer M, FitzGerald JM. A meta-analysis of the effect of Bacille Calmette Guerin vaccination on tuberculin skin test measurements. Thorax. 2002;57(9):804 PubMed -9.

20. Fjallbrant H, Ridell M, Larsson LO. BCG scar and tuberculin reactivity in children and adults. Scand J Infect Dis. 2008;40(5):387 PubMed -92.

21. Talati NJ, Seybold U, Humphrey B, Aina A, Tapia J, Weinfurter $\mathrm{P}$, et al. Poor concordance between interferon-gamma release assays and tuberculin skin tests in diagnosis of latent tuberculosis infection among HIV-infected individuals. BMC Infect Dis. 2009;9:15.

22. Farhat M, Greenaway C, Pai M, Menzies D. False-positive tuberculin skin tests: what is the absolute effect of BCG and non-tuberculous mycobacteria? Int J Tuberc Lung Dis. 2006;10(11):1192-204.
23. Addo K, van den Hof S, Mensah G, Hesse A, Bonsu C, Koram $\mathrm{K}$, et al. A tuberculin skin test survey among Ghanaian school children. BMC Public Health. 2010;10(1):35.

24. Rani SH, Vijayalakshmi V, Sunil K, Lakshmi KA, Suman LG, Murthy KJ. Cell mediated immunity in children with scar-failure following BCG vaccination. Indian Pediatr. 1998;35(2):123 PubMed -7.

25. Marcel D. Tuberculosis: a comprehensive international approach. 2nd edition. New York, 2000. p. 279 $-302$

26. Targeted tuberculin testing and treatment of latent tuberculosis infection. American Thoracic Society. MMWR Recomm Rep 2000;49:1-51 PubMed .

27. Long R, Houston S and Hershfield E. Recommendations for screening and prevention of tuberculosis in patients with HIV and for screening for HIV in patients with tuberculosis and their contacts. CMAJ 2003;169:789-91 PubMed

28. Musinguzi J, Kirungi W, Opio A, Montana L, Mishra V, Madraa E, et al. Comparison of HIV Prevalence Estimates From Sentinel Surveillance and a National Population-Based Survey in Uganda, 2004-2005. JAIDS Journal of Acquired Immune Deficiency Syndromes. 2009;51(1):78-84 10.1097/QAI.0b013e3181990713.

29. Brookmeyer R. Measuring the HIV/AIDS epidemic: approaches and challenges. Epidemiol Rev. 2010;32(1):26-37. 